\title{
Experiencing adversity - the importance of early detection and intervention
}

R. Campos ${ }^{1}$, AC. Serrano ${ }^{1}$, T. Cavaco ${ }^{1,}$ R. Esquina ${ }^{2}$

${ }^{1}$ Child and Adolescent Psychiatry Resident in Child and Adolescent Psychiatry Department of Hospital Garcia de Orta, Almada, Portugal

${ }^{2}$ Child and Adolescent Psychiatrist in Child and Adolescent Psychiatry Department of Hospital Garcia de Orta, Almada, Portugal

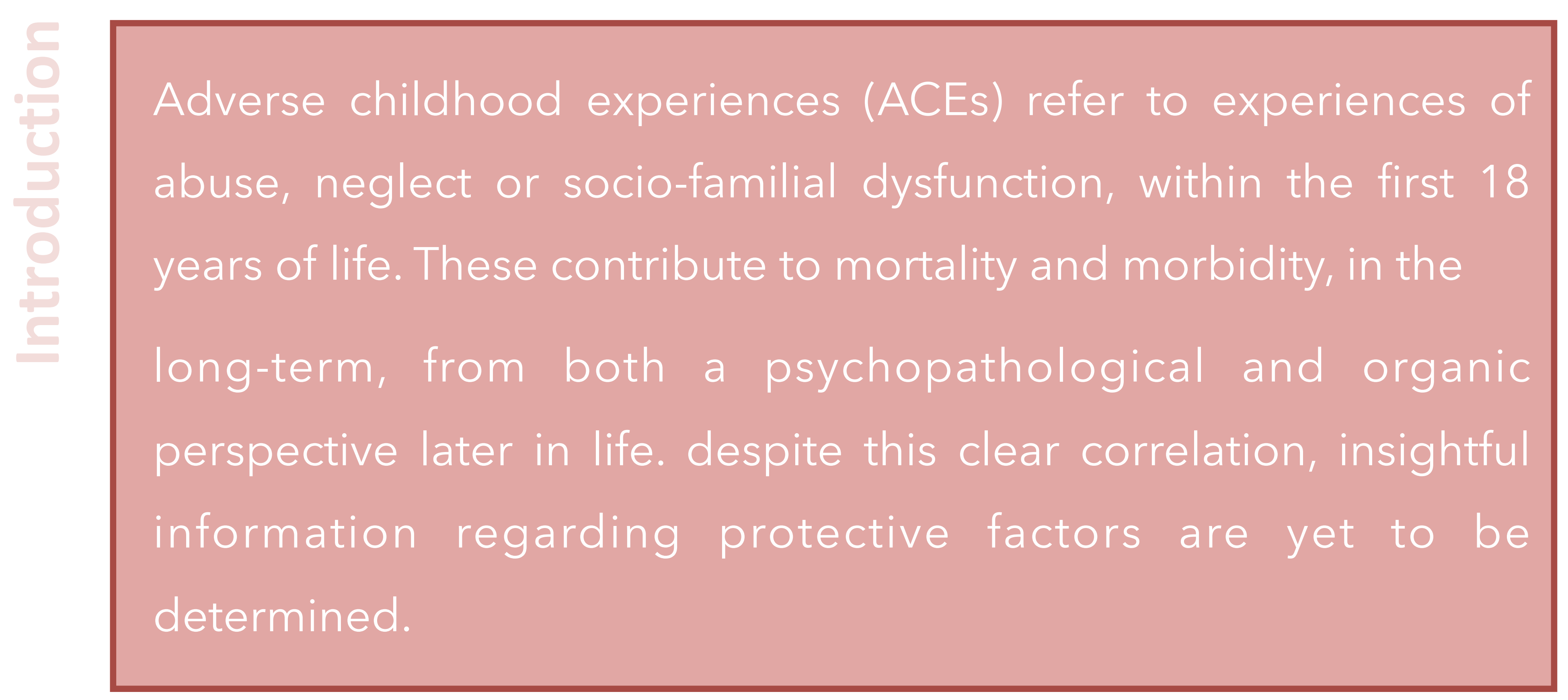

- Aim: Understanding the most prevalent psychopathology in adults exposed to ACEs, and the protective role of individual factors and of emotional and social support.

- Methods: Non-systematic review of the most recent literature, using Pubmed as research source.

\section{- Results}

- The most prevalent ACE are death of a parent, divorce, physical and sexual abuse and parental psychopathology, including addiction issues.

- Most frequently, individuals experience 2 or more ACEs simultaneously (40\%)

- Depressive disorder was identified as one of the most prevalent psychiatric diseases in adults with history of ACEs, followed by anxiety disorder and additive behaviours.
- 4 ou more ACEs increase the risk of alchool problems in $700 \%$; 6 or more ACEs are related to higher risk of suicide, in about $3000 \%$.

- The number of ACEs has a graded relationship to both lifetime and recent depressive disorders, suggesting that increased risk of depressive disorders remains up to decades after their occurrence.

- A positive correlation between the cumulative number of ACEs and the utilization of healthcare services was established, whereas contrarily, the presence of protective factors appear to inversely correlate with these events.

\section{Protective factors}

- Supportive family environment and social networks

- Concrete support for basic needs

- Nurturing parenting skills

- Stable family relationships

- Household rules and child monitoring

- Parental employment

- Parental education

- Adequate housing

- Access to healthcare and social services

- Caring adults outside the family who can serve as role models or mentors

- Communities that support parents and take responsibility for preventing abuse

- Child abuse and neglect and other ACEs are associated with a great impact on broader lifelong of both physical and mental health, increasing the risk of early death.

- Moreover, it is also related to poorer wellbeing outcomes, such as lower academic and employment opportunities, and greater rates of divorce.

- Providing safe, stable, nurturing relationships and environments can help preventing child abuse and neglect, and consequently negative adult life outcomes, such as mental health problems (e.g., depression).

- Considering depressive disorders are expected to become the second most common cause of death worldwide, an early intervention is important, in case of adverse experiences in childhood.

- Public health strategies and approaches must be implemented, in order to diminish the far-reaching consequences for the youngest and most vulnerable members of society, such as:

\footnotetext{
Strengthen economic support to families

Change social norms to support parents and positive parenting

Provide quality care and education early in life

Enhance parenting skills to promote healthy child development

Intervene to lessen harms and prevent future risk
} 